\title{
HALL EFFECTS AND ROTATION EFFECTS ON MHD FLOW PAST AN EXPONENTIALLY ACCELERATED VERTICAL PLATE WITH COMBINED HEAT AND MASS TRANSFER EFFECTS
}

\author{
M. THAMIZHSUDAR ${ }^{*}$ and J. PANDURANGAN \\ Department of Mathematics \\ Aarupadai Veedu Institute of Technology \\ Paiyanoor-603 104, Chennai, INDIA \\ E-mail: thamizhsudar80@gmail.com \\ R. MUTHUCUMARASWAMY \\ Department of Applied Mathematics \\ Sri Venkateswara College of Engineering \\ Sriperumbudur - 602 105, INDIA
}

\begin{abstract}
A theoretical solution of flow past an exponentially accelerated vertical plate in the presence of Hall current and MHD relative to a rotating fluid with uniform temperature and mass diffusion is presented. The dimensionless equations are solved using the Laplace method. The axial and transverse velocity, temperature and concentration fields are studied for different parameters such as the Hall parameter (m), Hartmann number (M), Rotation parameter $(\Omega)$, Schmidt number, Prandtl number, thermal Grashof number (Gr) and mass Grashof number $(\mathrm{Gc})$. It has been observed that the temperature of the plate decreases with increasing values of the Prandtl number and the concentration near the plate increases with decreasing values of Schmidt number. It is also observed that both axial and transverse velocities increase with decreasing values of the magnetic field parameter or rotation parameter, but the trend gets reversed with respect to the Hall parameter. The effects of parameters $\mathrm{m}, \mathrm{M}, \Omega, \mathrm{Gr}$ and $\mathrm{Gc}$ on the axial and transverse velocity profiles are shown graphically.
\end{abstract}

Key words: Hall effect, MHD flow, rotation, exponentially, accelerated plate, temperature, mass diffusion.

\section{Introduction}

The influence of the magnetic field on a viscous incompressible flow of an electrically conducting fluid has its importance in many applications such as geophysics, metallurgy and aerodynamics, extrusion of plastics in the manufacture of rayon, nylon, purification of crude oil and other engineering processes such as in petroleum engineering, chemical engineering, composite or ceramic engineering and heat exchangers.

The rotating flow of an electrically conducting fluid in the presence of a magnetic fluid is encountered in cosmical, geophysical fluid dynamics. Also in solar physics involved in the sunspot development, the solar cycle and the structure of rotating magnetic stars. The study of MHD viscous flows with Hall currents has important engineering applications in problems of MHD generators, Hall accelerators as well as in flight magneto hydrodynamics.

The effect of Hall currents on a hydromagnetic flow near an accelerated plate was studied by Pop (1971). Rotation effects on a hydromagnetic free convective flow past an accelerated isothermal vertical plate were studied by Raptis and Singh (1981). Takhar et al. (1992) studied the Hall effects on heat and mass transfer flow with variable suction and heat generation. Watnab and Pop (1995) studied the effect of Hall current on the steady MHD flow over a continuously moving plate, when the liquid is permeated by a

\footnotetext{
* To whom correspondence should be addressed
} 
uniform transverse magnetic field. Takhar et al. (2002) investigated the simultaneous effects of Hall current and free stream velocity on the magneto hydrodynamic flow over a moving plate in a rotating fluid. Hayat and Abbas (2007) studied the fluctuating rotating flow of a second-grade fluid past a porous plate with variable suction and Hall current. Muthucumaraswamy et al. (2008) obtained the heat transfer effects on flow past an exponentially accelerated vertical plate with variable temperature. Rotation effects on an MHD flow past an accelerated plate with variable temperature and uniform mass diffusion were studied by Muthucumaraswamy and Tinalal (2011). Magua and Mutua (2013) studied the Hall current effects on free convection flow and mass transfer past a semi-infinite vertical flat plate.

In all the above studies, the combined effect of rotation and MHD flow in addition to Hall current has not been considered simultaneously. Here we have made an attempt to study the Hall current effects on a MHD flow of an exponentially accelerated horizontal plate relative to a rotating fluid with uniform temperature and mass diffusion.

\section{Mathematical formulation}

Here we consider an electrically conducting viscous incompressible fluid past an infinite plate occupying the plane $z^{\prime}=0$. The $x^{\prime}$-axis is taken in the direction of the motion of the plate and $y^{\prime}$-axis is normal to both $x^{\prime}$ and $z^{\prime}$ axes. Initially, the fluid and the plate rotate in unison with a uniform angular velocity $\Omega^{\prime}$ about the $z^{\prime}$-axis normal to the plate, also the temperature of the plate and concentration near the plate are assumed to be $T_{\infty}$ and $c_{\infty}^{\prime}$. At time $t^{\prime}>0$, the plate is exponentially accelerated with a velocity $u^{\prime}=\frac{u_{0}}{A} \exp \left(a^{\prime} t^{\prime}\right)$ in its own plane along the $x^{\prime}$-axis and the temperature near the plate is raised to $T_{w}$ and the concentration level near the plate is also raised to $c_{w}^{\prime}$. Here the plate is electrically non conducting. Also, a uniform magnetic field $B_{0}$ is applied parallel to the $z^{\prime}$-axis. Also the pressure is uniform in the flow field. If $u^{\prime}, v^{\prime}, w^{\prime}$ represent the components of the velocity vector $F$, then the equation of continuity $\nabla \cdot F=0$ gives $w^{\prime}=0$ everywhere in the flow such that the boundary condition $w^{\prime}=0$ is satisfied at the plate. Here the flow quantities depend on $z^{\prime}$ and $t^{\prime}$ only and it is assumed that the flow far away from the plate is undisturbed. Under these assumptions the unsteady flow is governed by the following equations.

$$
\begin{aligned}
& \frac{\partial u^{\prime}}{\partial t^{\prime}}=v \frac{\partial^{2} u^{\prime}}{\partial z^{\prime 2}}+2 \Omega^{\prime} v^{\prime}-\frac{\sigma \mu_{e}^{2} B_{0}^{2}}{\rho\left(1+m^{2}\right)}\left(u^{\prime}+m v^{\prime}\right)+g \beta\left(T-T_{\infty}\right)+g \beta *\left(c^{\prime}-c_{\infty}^{\prime}\right), \\
& \frac{\partial v^{\prime}}{\partial t^{\prime}}=v \frac{\partial^{2} v^{\prime}}{\partial z^{\prime 2}}-2 \Omega^{\prime} u^{\prime}+\frac{\sigma \mu_{e}^{2} B_{0}^{2}}{\rho\left(1+m^{2}\right)}\left(m u^{\prime}-v^{\prime}\right) \\
& \rho c_{p} \frac{\partial T}{\partial t^{\prime}}=K \frac{\partial^{2} T}{\partial z^{\prime 2}} \\
& \frac{\partial c^{\prime}}{\partial t^{\prime}}=D \frac{\partial^{2} c^{\prime}}{\partial z^{\prime 2}}
\end{aligned}
$$

where $u^{\prime}$ is the axial velocity and $v^{\prime}$ is the transverse velocity. The prescribed initial and boundary conditions are

$$
u^{\prime}=0, \quad T=T_{\infty}, \quad c^{\prime}=c_{\infty}^{\prime} \quad \text { at } \quad t^{\prime} \leq 0 \quad \text { for all } \quad z^{\prime},
$$




$$
\begin{aligned}
& u^{\prime}=\frac{u_{0}}{A} e^{a^{\prime} t^{\prime}}, \quad v^{\prime}=0, \quad T=T_{\mathrm{w}}, \quad c^{\prime}=c_{w}^{\prime} \quad \text { at } \quad z^{\prime}=0 \quad f \quad \text { or all } \quad t^{\prime}>0, \\
& u^{\prime} \rightarrow 0, \quad v^{\prime} \rightarrow 0, \quad T \rightarrow T_{\infty}, \quad c^{\prime} \rightarrow c_{\infty}^{\prime} \quad \text { as } \quad z^{\prime} \rightarrow \infty
\end{aligned}
$$

where, $A=\left(\frac{u_{0}^{2}}{v}\right)^{\frac{1}{3}}$ is a constant.

On introducing the following non-dimensional quantities

$$
\begin{aligned}
& u=\frac{u^{\prime}}{\left(u_{0} \mathrm{v}\right)^{\frac{1}{3}}}, \quad v=\frac{v^{\prime}}{\left(u_{0} \mathrm{v}\right)^{\frac{1}{3}}}, \quad z=z^{\prime}\left(\frac{u_{0}}{v^{2}}\right)^{\frac{1}{3}}, \quad t=\left(\frac{u_{0}^{2}}{v}\right)^{\frac{1}{3}} t^{\prime}, \quad \Omega=\Omega^{\prime}\left(\frac{v}{u_{0}^{2}}\right)^{\frac{1}{3}}, \\
& M^{2}=\frac{\sigma \mu_{e}^{2} B_{0}^{2} v^{\frac{1}{3}}}{2 \rho u_{0}^{2 / 3}}, \quad a=\left(\frac{v}{u_{0}^{2}}\right)^{\frac{1}{3}} a^{\prime}, \quad \mathrm{Gc}=\frac{g \beta^{*}\left(c_{w}^{\prime}-c_{\infty}^{\prime}\right)}{u_{0}}, \quad \mathrm{Gr}=\frac{g \beta\left(T_{w}-T_{\infty}\right)}{u_{0}}, \\
& C=\frac{c^{\prime}-c_{\infty}^{\prime}}{c_{w}^{\prime}-c_{\infty}^{\prime}}, \quad \theta=\frac{T-T_{\infty}}{T_{w}-T_{\infty}}, \quad \operatorname{Pr}=\frac{\mu c_{p}}{K}, \quad \mathrm{Sc}=\frac{v}{D} .
\end{aligned}
$$

Equations (2.1)-(2.7) reduce to the following non-dimensional form of governing equations

$$
\begin{aligned}
& \frac{\partial u}{\partial t}=\frac{\partial^{2} u}{\partial z^{2}}+2 \Omega v-\frac{2 M^{2}}{1+m^{2}}(u+m v)+\operatorname{Gr} \theta+\operatorname{Gc} C, \\
& \frac{\partial v}{\partial t}=\frac{\partial^{2} v}{\partial z^{2}}-2 \Omega u+\frac{2 M^{2}}{1+m^{2}}(m u-v), \\
& \frac{\partial \theta}{\partial t}=\frac{1}{\operatorname{Pr}} \frac{\partial^{2} \theta}{\partial z^{2}}, \\
& \frac{\partial C}{\partial t}=\frac{1}{\operatorname{Sc}} \frac{\partial^{2} C}{\partial z^{2}},
\end{aligned}
$$

with initial and boundary conditions

$$
\begin{aligned}
& u=0, \quad v=0, \quad \theta=0, \quad C=0 \quad \text { at } \quad t \leq 0 \quad \text { for all } \quad z, \\
& u=e^{a t}, \quad v=0, \quad \theta=1, \quad C=1 \quad \text { at } \quad t>0, \quad z=0, \\
& u \rightarrow 0, \quad v \rightarrow 0, \quad \theta \rightarrow 0, \quad C \rightarrow 0 \quad \text { as } \quad z \rightarrow \infty .
\end{aligned}
$$

The above Eqs (2.8) - (2.9) and boundary conditions (2.12)-(2.14) can be combined as

$$
\frac{\partial q}{\partial t}=\frac{\partial^{2} q}{\partial z^{2}}-2 q\left[\frac{M^{2}}{1+m^{2}}+i\left(\Omega-\frac{M^{2} m}{1+m^{2}}\right)\right]+\operatorname{Gr} \theta+\operatorname{Gc} C,
$$




$$
\begin{aligned}
& \frac{\partial \theta}{\partial t}=\frac{1}{\operatorname{Pr}} \frac{\partial^{2} \theta}{\partial z^{2}}, \\
& \frac{\partial C}{\partial t}=\frac{1}{\operatorname{Sc}} \frac{\partial^{2} C}{\partial Z^{2}},
\end{aligned}
$$

with boundary conditions

$$
\begin{aligned}
& q=0, \quad \theta=0, \quad C=0 \quad \text { at } \quad t \leq 0 \quad \text { for all } \quad z, \\
& q=e^{a t}, \quad \theta=1, \quad C=1 \quad \text { at } \quad z=0, \quad \text { for all } \quad t>0, \\
& q \rightarrow 0, \quad \theta \rightarrow 0, \quad C \rightarrow 0 \quad \text { as } \quad z \rightarrow \infty
\end{aligned}
$$

where $q=u+i v$.

\section{Solution of the problem.}

To solve the dimensionless governing Eqs (2.15) to (2.17), subject to the initial and boundary conditions (2.18)-(2.20) the Laplace transform technique is used. The solutions are in terms of exponential and complementary error functions

$$
\begin{aligned}
& C=\operatorname{erfc}(\eta \sqrt{\mathrm{Sc}}), \\
& \theta=\operatorname{erfc}(\eta \sqrt{\operatorname{Pr}}) \\
& q=\frac{e^{a t}}{2}\left[\begin{array}{l}
\exp (2 \eta \sqrt{(a+b) t} \operatorname{erfc}(\eta+\sqrt{(a+b) t})+ \\
+\exp (-2 \eta \sqrt{(a+b) t} \operatorname{erfc}(\eta-\sqrt{(a+b) t})
\end{array}\right]+ \\
& -\frac{1}{2}\left[\frac{c}{d}+\frac{e}{f}\right]\left[\begin{array}{c}
\exp (-2 \eta \sqrt{b t}) \operatorname{erfc}(\eta-\sqrt{b t})+ \\
+\exp (2 \eta \sqrt{b t}) \operatorname{erfc}(\eta+\sqrt{b t})
\end{array}\right]+ \\
& +\frac{c}{d}[(\exp (d t) / 2)]\left[\begin{array}{l}
\exp (2 \eta \sqrt{(b+d) t}) \operatorname{erfc}(\eta+\sqrt{(b+d) t})+ \\
+\exp (-2 \eta \sqrt{(b+d) t}) \operatorname{erfc}(\eta-\sqrt{(b+d) t})
\end{array}\right]+ \\
& +\frac{e}{f}\left[\left[\frac{\exp (f t)}{2}\right]\left[\begin{array}{l}
\exp (2 \eta \sqrt{(b+f) t}) \operatorname{erfc}(\eta+\sqrt{(b+f) t})+ \\
+\exp (-2 \eta \sqrt{(b+f) t}) \operatorname{erfc}(\eta-\sqrt{(b+f) t})
\end{array}\right]\right]+\frac{c}{d}[\operatorname{erfc}(\eta \sqrt{\operatorname{Pr}})]+ \\
& -\frac{c}{d}\left[\frac{\exp (d t)}{2}\right]\left[\begin{array}{c}
\exp (-2 \eta \sqrt{\operatorname{Pr} d t}) \operatorname{erfc}(\eta \sqrt{\operatorname{Pr}}-\sqrt{d t})+ \\
+\exp (2 \eta \sqrt{\operatorname{Pr} d t}) \operatorname{erfc}(\eta \sqrt{\operatorname{Pr}}+\sqrt{d t})
\end{array}\right]+\frac{e}{f}[\operatorname{erfc}(\eta \sqrt{\operatorname{Sc}})]+ \\
& -\frac{e}{f}\left[\frac{\exp (f t)}{2}\right]\left[\begin{array}{l}
\exp (-2 \eta \sqrt{\operatorname{Sc} f t}) \operatorname{erfc}(\eta \sqrt{\mathrm{Sc}}-\sqrt{f t})+ \\
+\exp (2 \eta \sqrt{\operatorname{Sc} f t}) \operatorname{erfc}(\eta \sqrt{\mathrm{Sc}}+\sqrt{f t})
\end{array}\right]
\end{aligned}
$$


where

$$
\begin{aligned}
& b=2\left[\frac{M^{2}}{1+m^{2}}+i\left(\Omega-\frac{M^{2} m}{1+m^{2}}\right)\right], \quad c=\frac{\mathrm{Gr}}{\mathrm{Pr}-1}, \quad d=\frac{b}{\mathrm{Pr}-1}, \\
& e=\frac{\mathrm{Gc}}{\mathrm{Sc}-1}, \quad f=\frac{b}{\mathrm{Sc}-1}, \quad \eta=z / 2 \sqrt{t} .
\end{aligned}
$$

In order to get a clear understanding of the flow field, we have separated $q$ into real and imaginary parts to obtain axial and transverse components $u$ and $v$.

\section{Results and discussion}

To interpret the results for a better understanding of the problem, numerical calculations are carried out for different physical parameters $M, m, \Omega, \mathrm{Gr}, \mathrm{Gc}$, Pr and Sc. The value of the Prandtl number is chosen to be 7.0 which represents water.

Figure 1 illustrates the effect of the Schmidt number ( $\mathrm{Sc}=0.16,0.3,0.6$ ), $M=m=0.5, \Omega=0.1, a=2.0$, $t=0.2$ on the concentration field. It is observed that, as the Schmidt number increases, the concentration of the fluid medium decreases. The effect of the Prandtl number (Pr) on the temperature field is shown in Fig.2. It is noticed that an increase in the Prandtl number leads to a decrease in the temperature.

Figure 3 illustrates that effects of the magnetic field parameter $(M=0.5,3.0,5.0), \mathrm{Gr}=\mathrm{Gc}=5.0$, $m=0.5, a=2.0, t=0.2)$ on axial velocity. It is observed that the axial velocity increases with the decreasing value of $M$. This shows that the increase in the magnetic field parameter leads to a fall in velocity. This agrees with the expectations, since the magnetic field exerts a retarding force on the free convective flow.

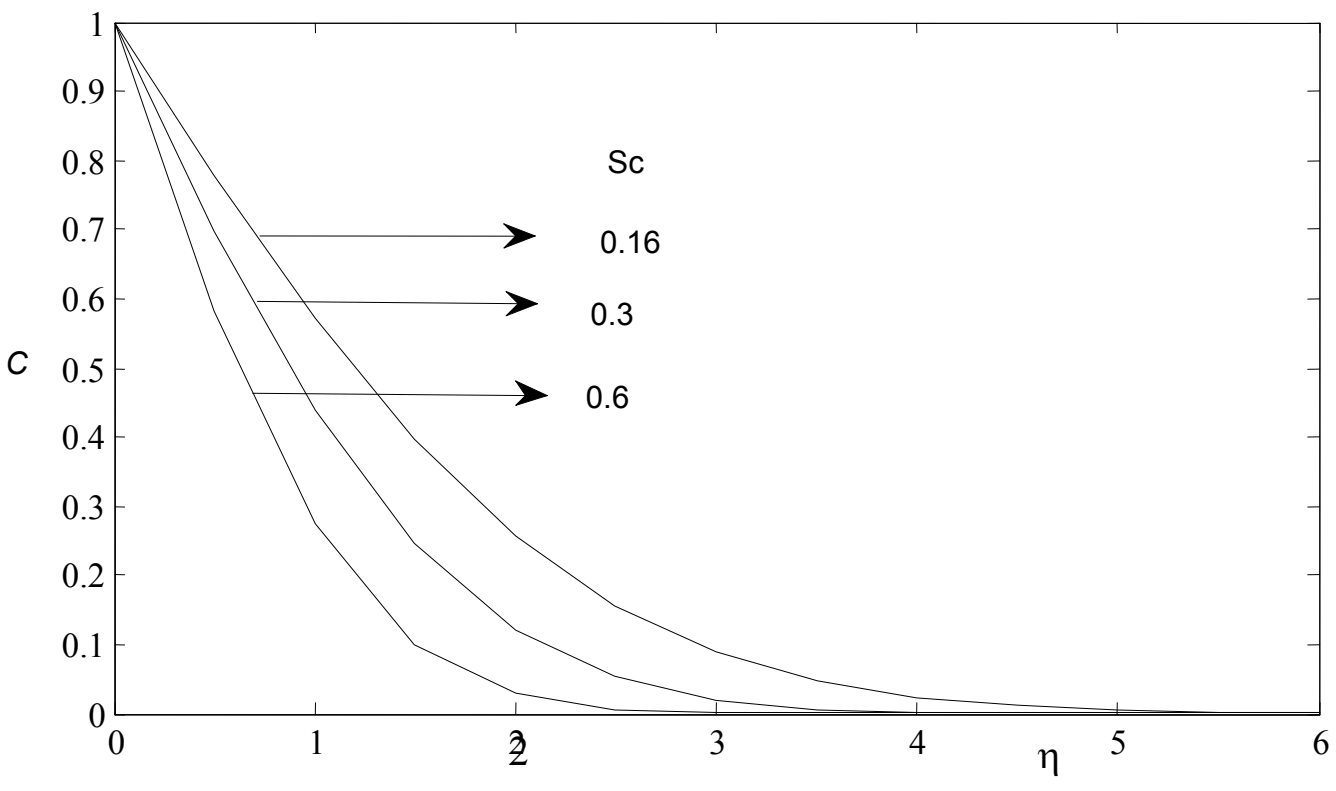

Fig.1. Concentration profiles for different values of Sc. 


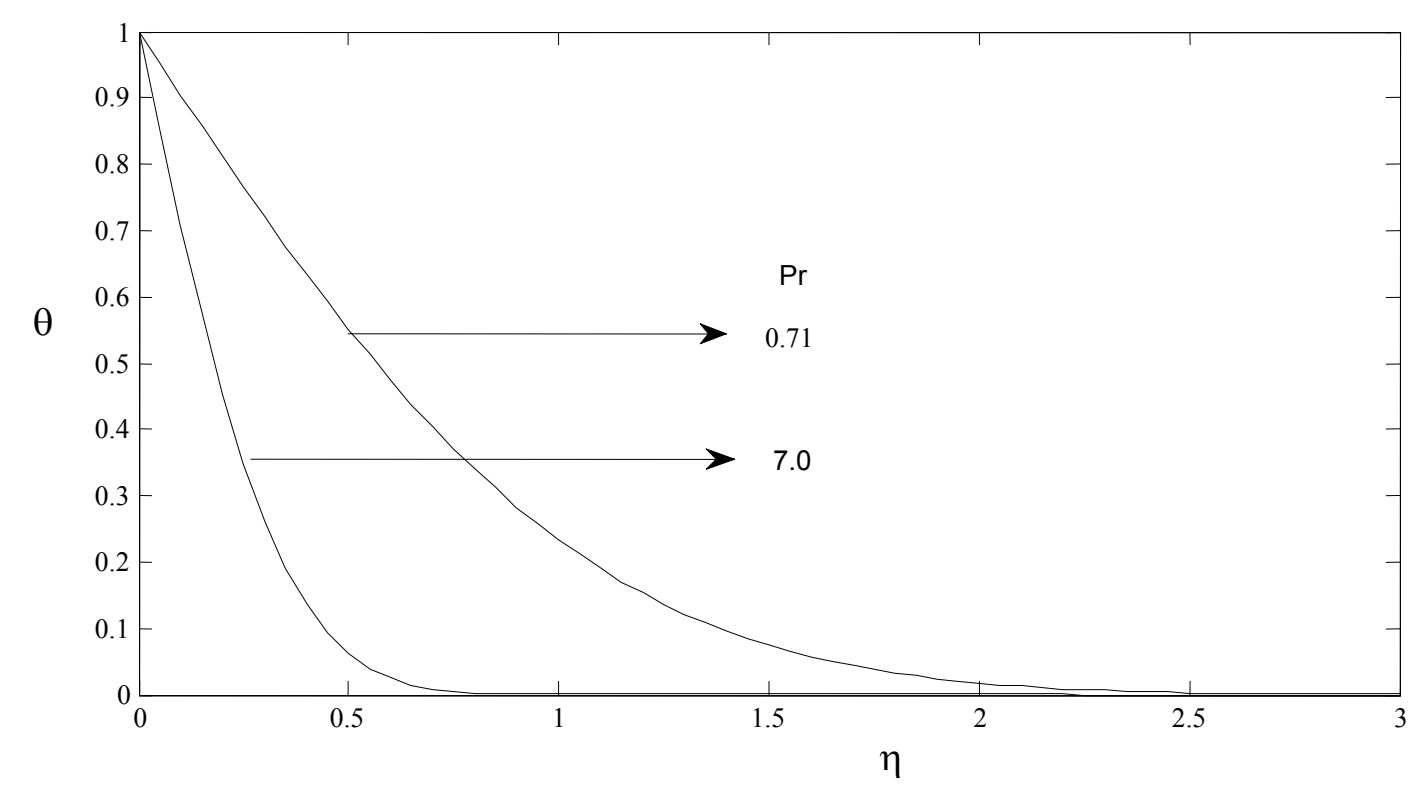

Fig.2. Temperature profiles for different values of Pr.

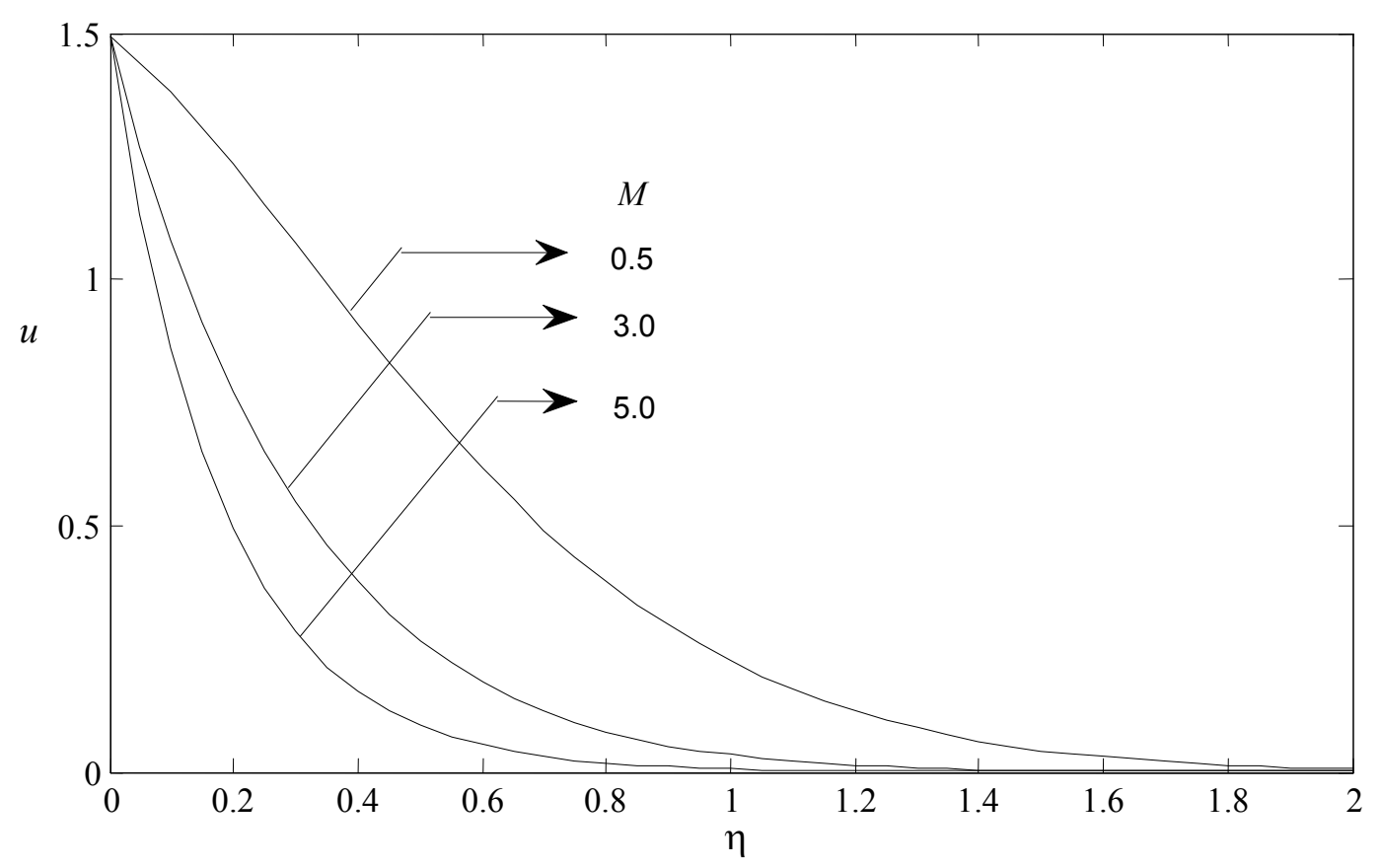

Fig.3. Axial velocity profiles for different values of $M$.

The effect of the rotation parameter on axial velocity is shown in Fig.4. It is observed that the velocity increases with decreasing values of $\Omega$ form 0.1 to 0.5 . It is also found that when the rotation parameter increases beyond 0.5 , the effect lies within the given range 0.1 to 0.5 . 


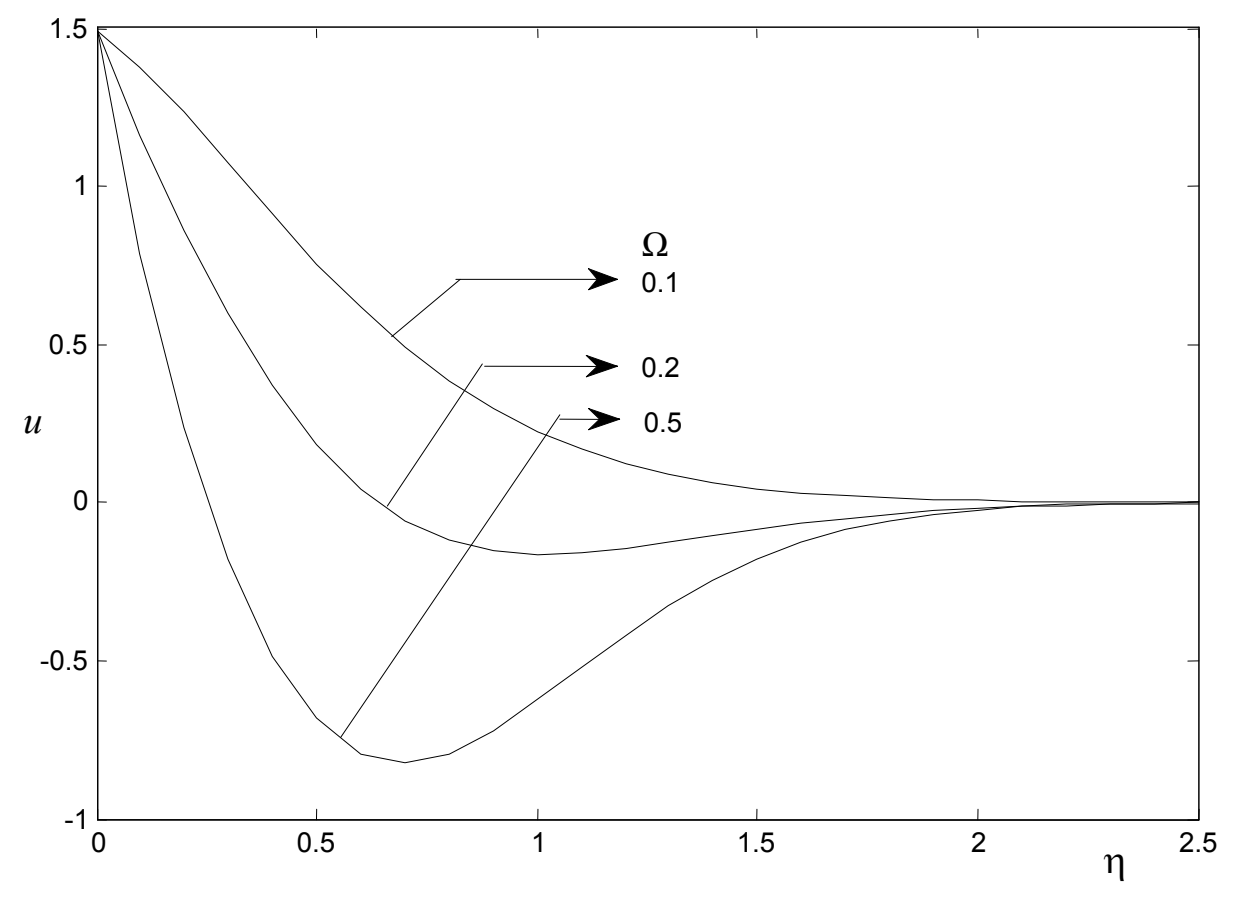

Fig.4. Axial velocity profiles for different values of $\Omega$.

Figure 5 demonstrates the effect of the Hall parameter $m$ on axial velocity. It has been noticed that the velocity increases with increasing values of the Hall parameter.

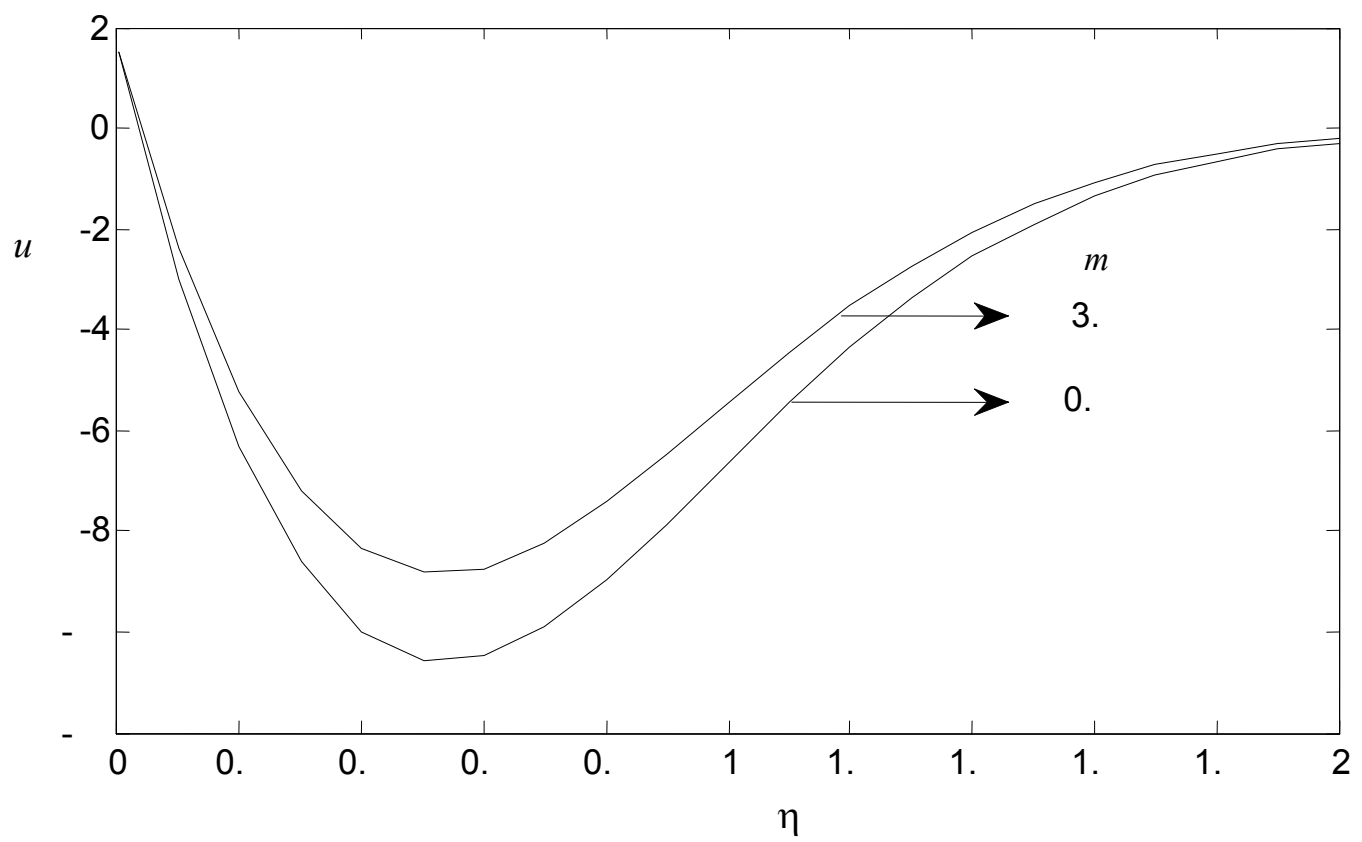

Fig.5. Axial velocity profiles for different values of $m$. 
Figures 6 and 7 show the effects of the thermal Grashof number Gr and mass Grashof number Gc. It has been noticed that the velocity increases with increasing values of both $\mathrm{Gr}$ and $\mathrm{Gc}$, but the effect seems to be meager in both the cases.

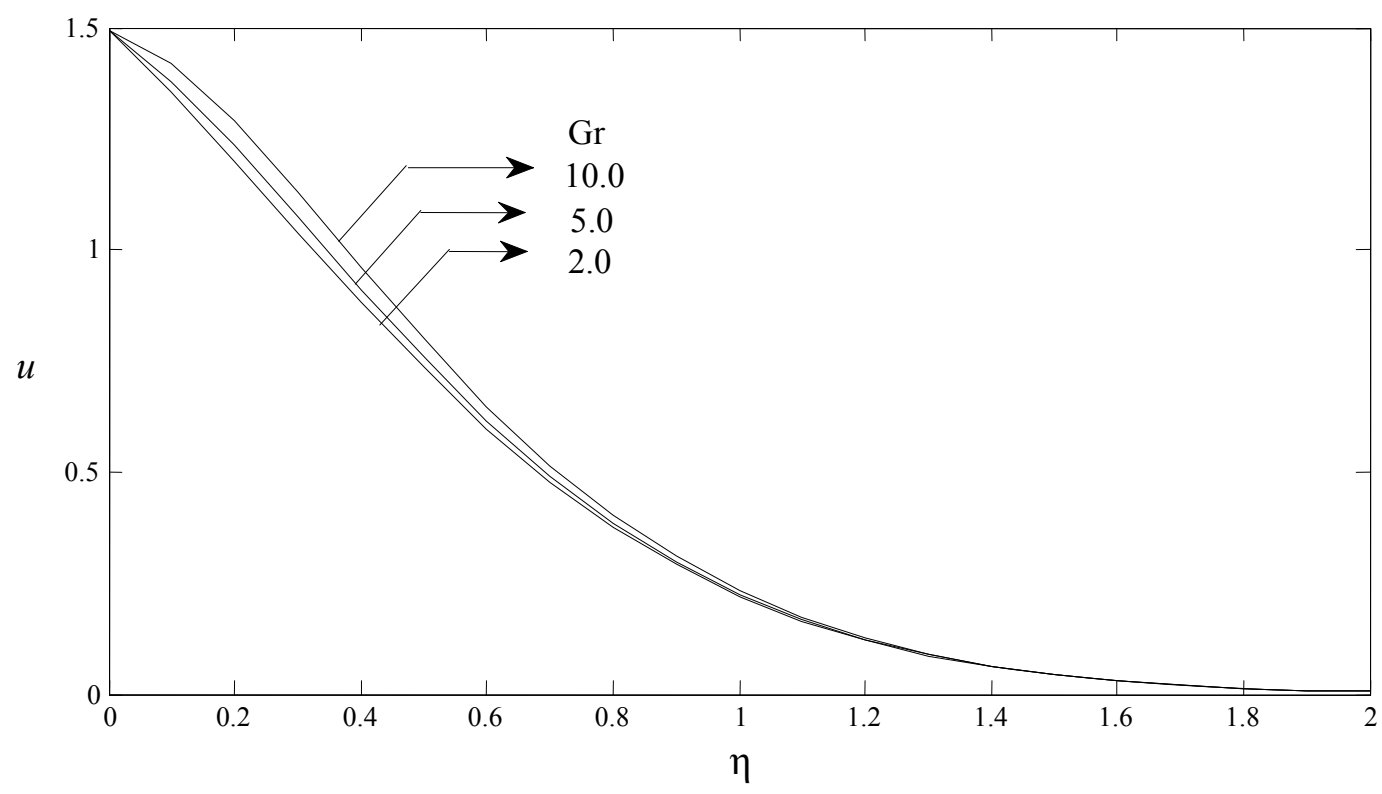

Fig.6. Axial velocity profiles for different values of Gr.

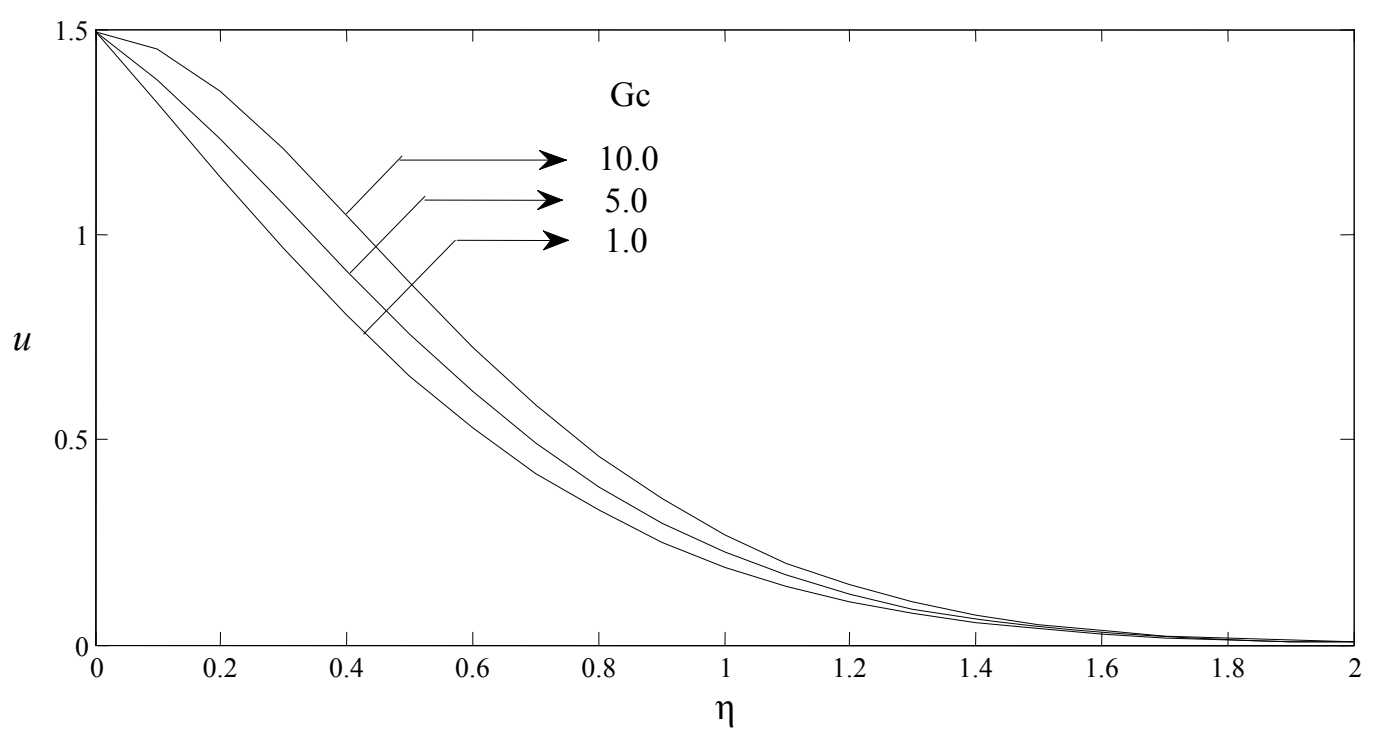

Fig.7. Axial velocity profiles for different values of Gc.

Figure 8 illustrates the effects of the magnetic field parameter $M$ on transverse velocity. It is observed that the transverse velocity increases with decreasing values of $M$ as in the case of axial velocity. 


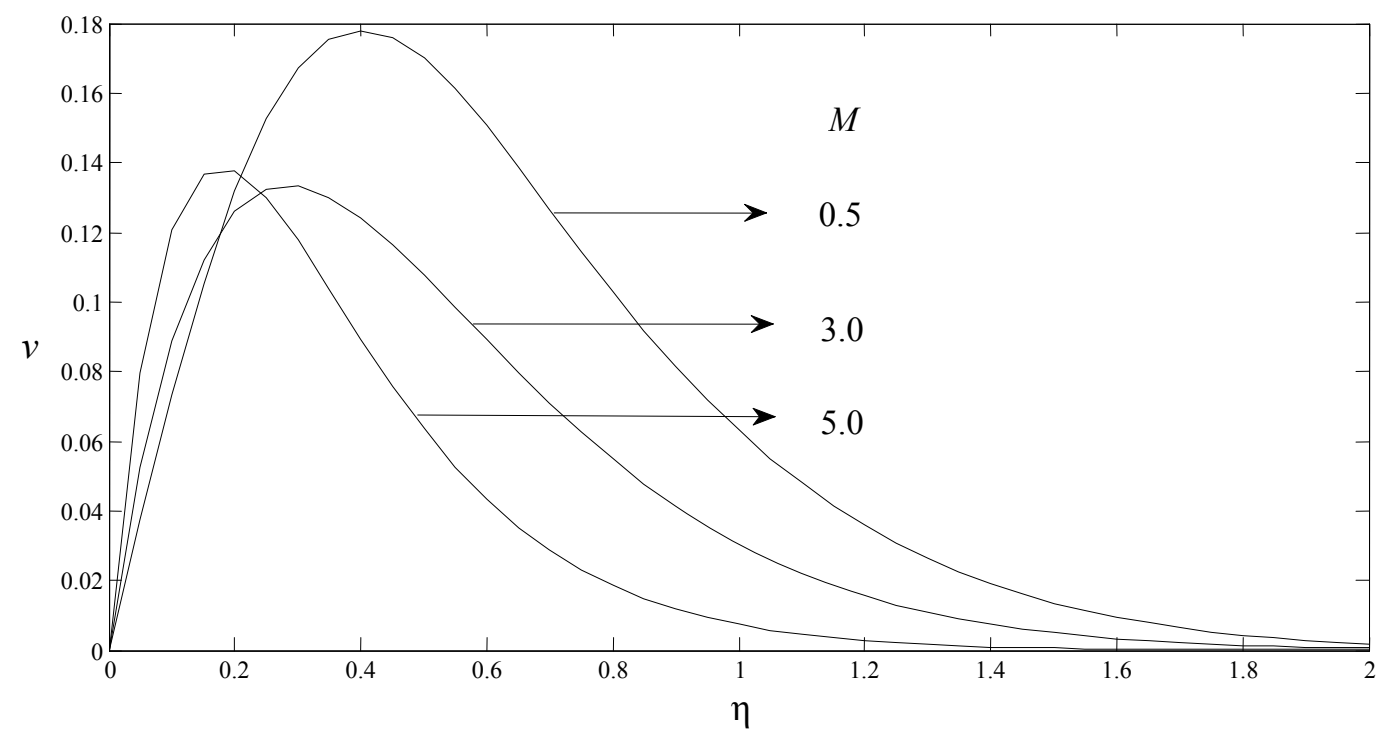

Fig.8. Transverse velocity profiles for different values of $M$.

The transverse velocity profiles for different values of the rotation parameter $\Omega$ are shown in Fig.9. It is observed that the velocity increases with decreasing values of $\Omega$.

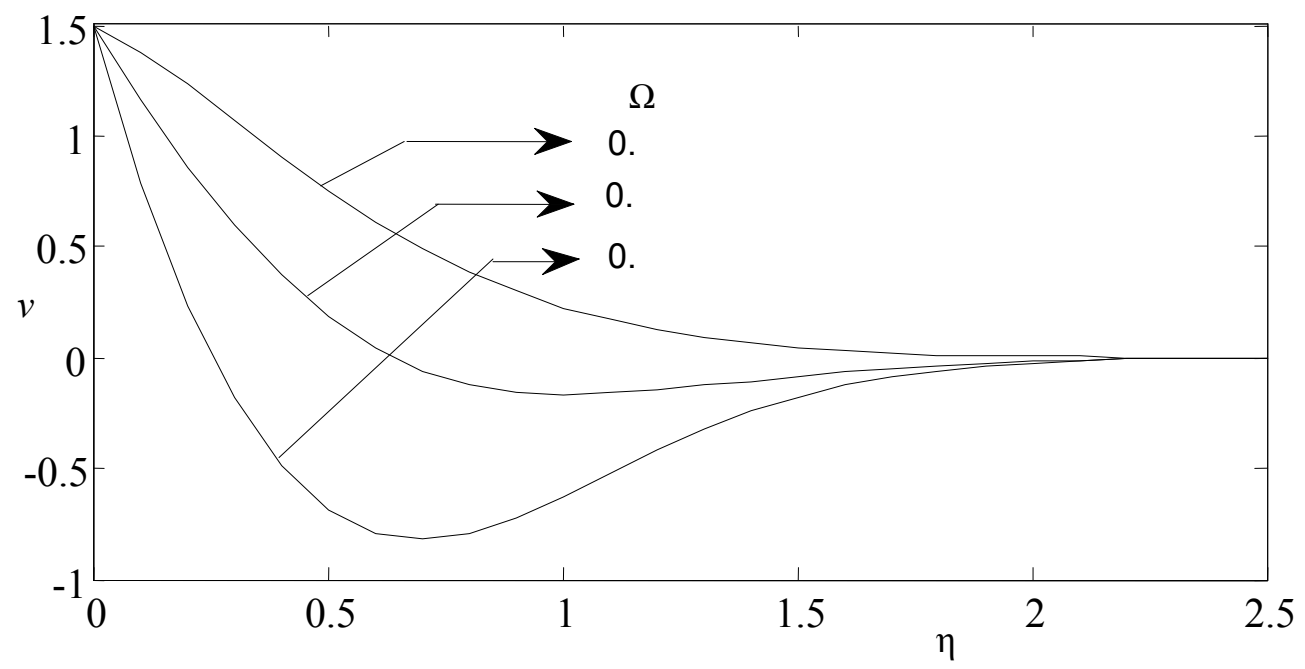

Fig.9. Transverse velocity profiles for different values of $\Omega$.

Figure 10 shows the effect of the Hall parameter $m$ on transverse velocity. It is found that the velocity increases with increasing values of $m$, but when the Hall parameter takes values beyond 2.0, there is a sudden fall in transverse velocity.

Figure 11 demonstrates the effect of the thermal Grashof number Gr on transverse velocity. It is observed that there is an increase in velocity as there is an increase in Gr. 
The effect of the mass Grashof number on transverse velocity is shown in Fig.12. Numerical calculations were carried out for different values of Gc, namely: $0,5,10,20$. Since the curve remains same for all these values, only two values of Gc $(0$ and 5$)$ are taken to be described graphically. From the figure it has been noticed that with increasing values of Gc the transverse velocity is unaffected.

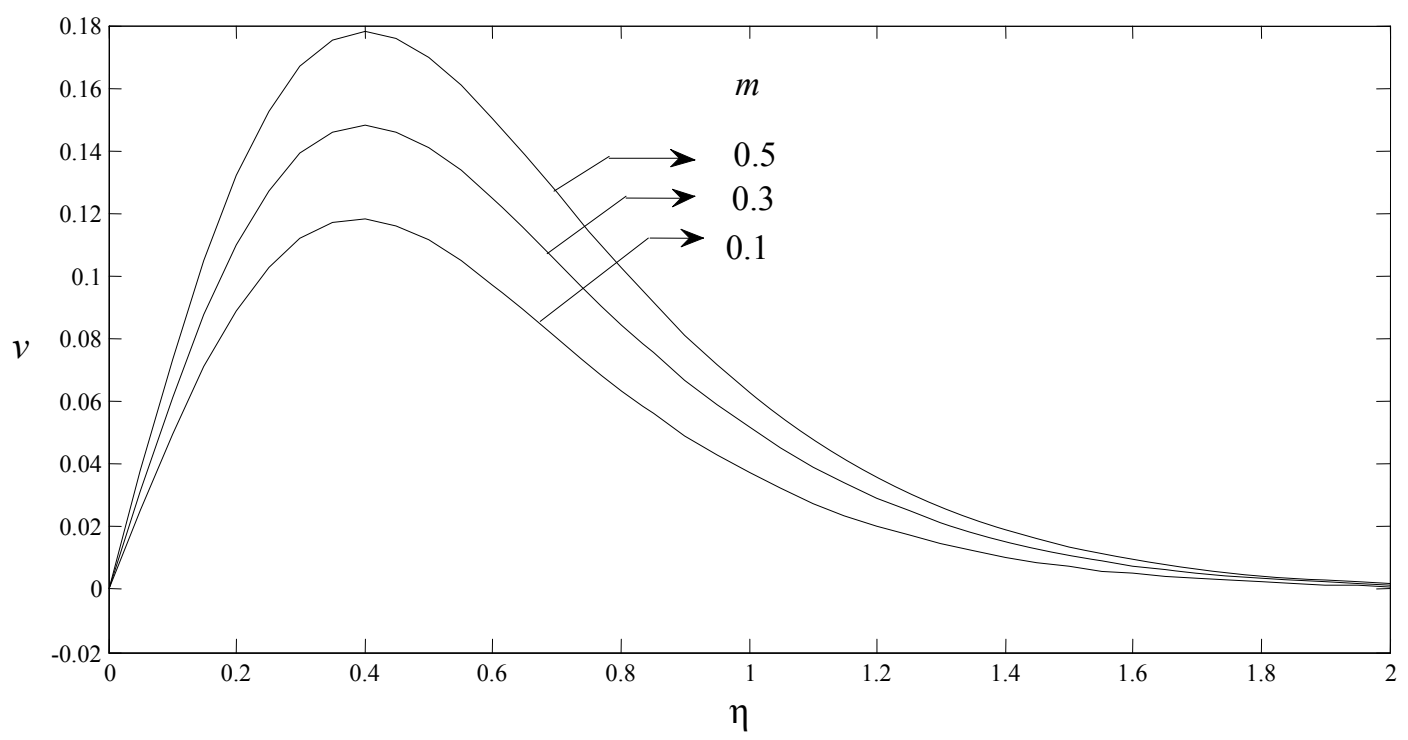

Fig.10. Transverse velocity profiles for different values of $m$.

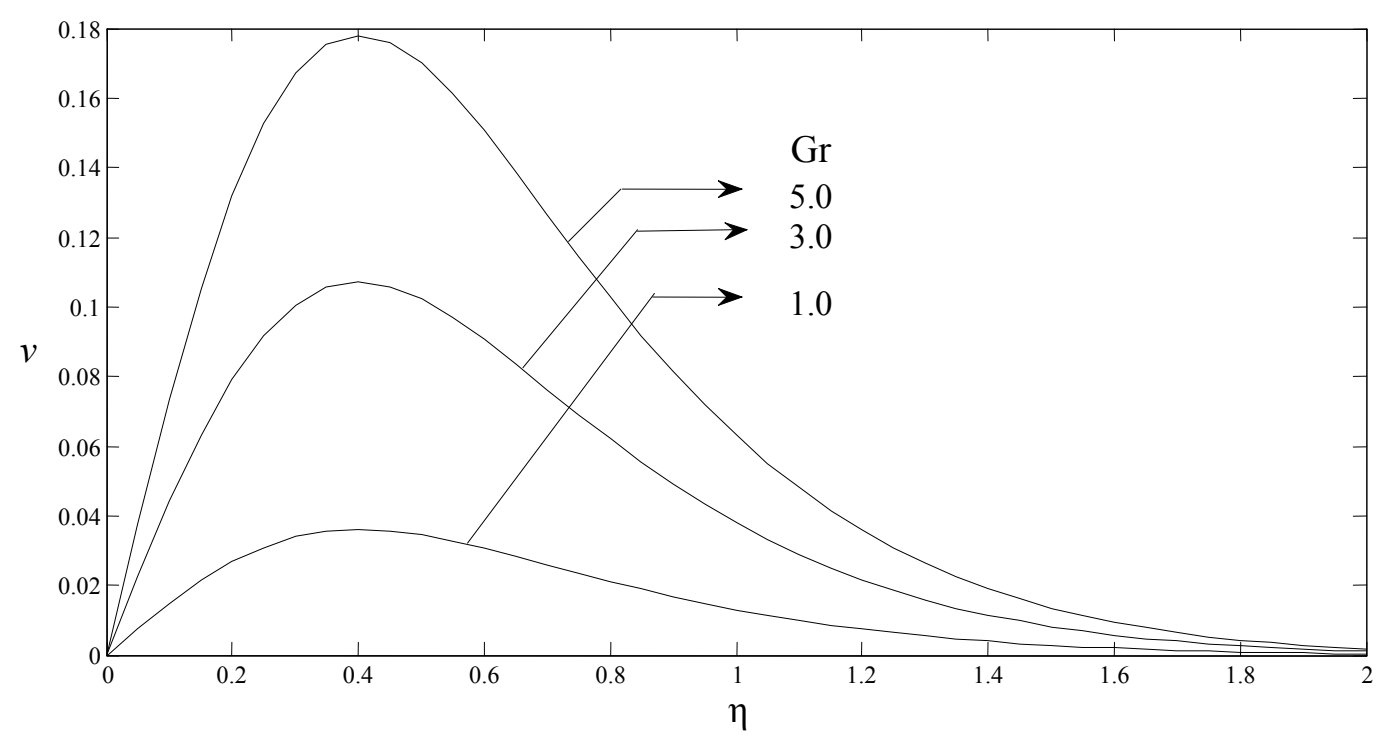

Fig.11. Transverse velocity profiles for different values of Gr. 


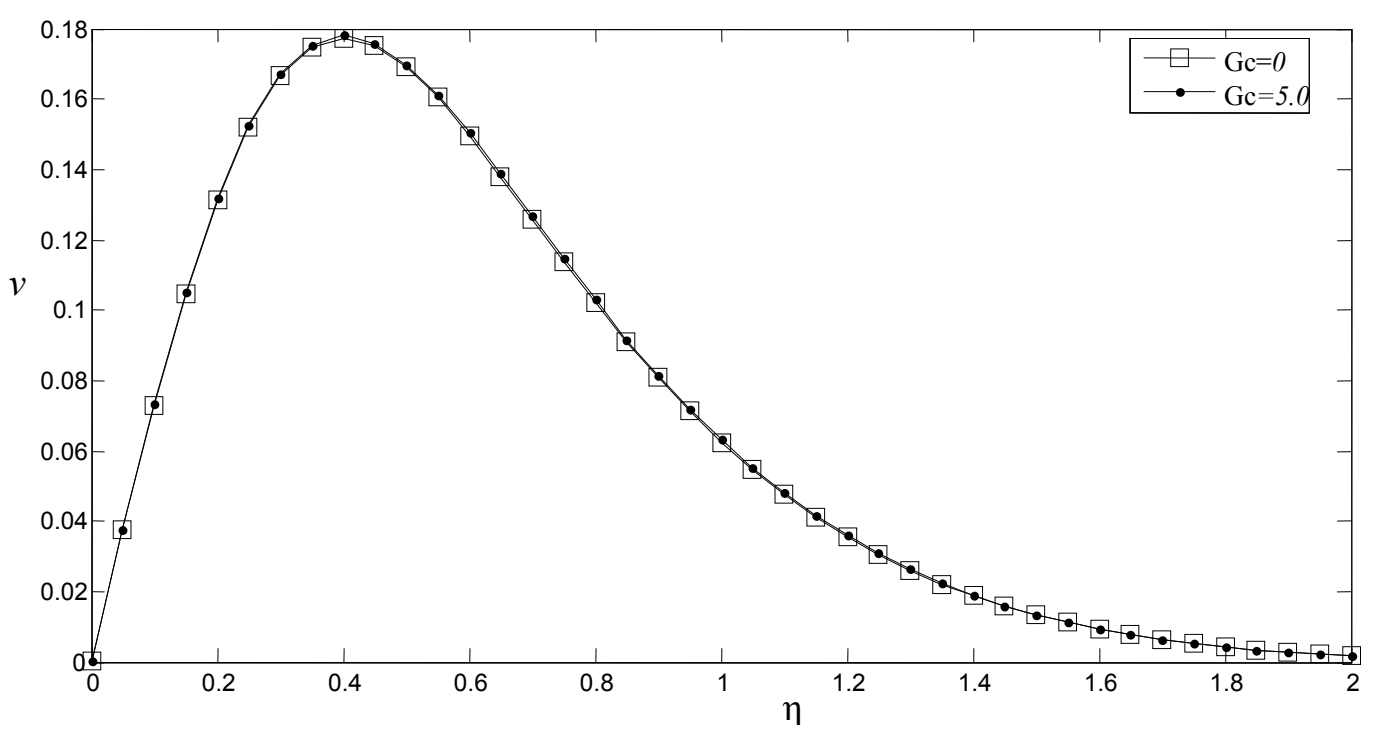

Fig.12. Transverse velocity profiles for different values of Gc.

\section{Conclusion}

In this paper we have studied the effects of Hall current, rotation effect on an MHD flow through an exponentially accelerated vertical plate with uniform temperature and mass diffusion. In the analysis of the flow the following conclusions are made.

(1) The temperature of the plate decreases with increasing values of the Prandtl number.

(2) The concentration near the plate increases with decreasing values of the Schmidt number.

(3) Both axial and transverse velocities increase with decreasing values of the magnetic field parameter or rotation parameter, but the trend gets reversed with respect to the Hall parameter.

(4) Both the velocities increase with increasing values of the thermal Grashof number but the effect is seen to be meager on axial velocity compared with change in transverse velocity.

(5) The effect of the mass Grashof number on axial velocity is seen to be meager whereas the effect is almost nil on transverse velocity.

\section{Nomenclature}

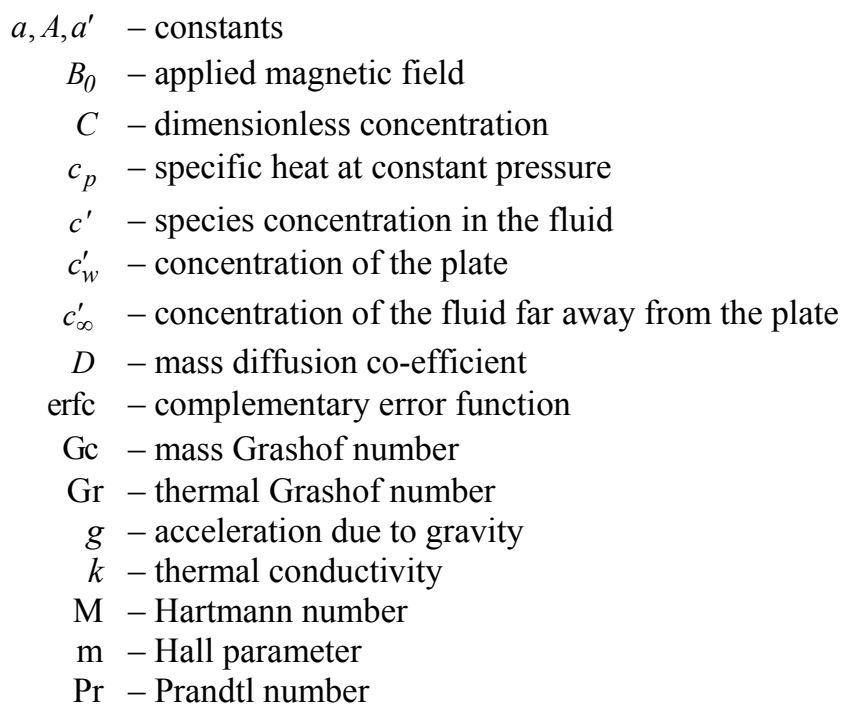




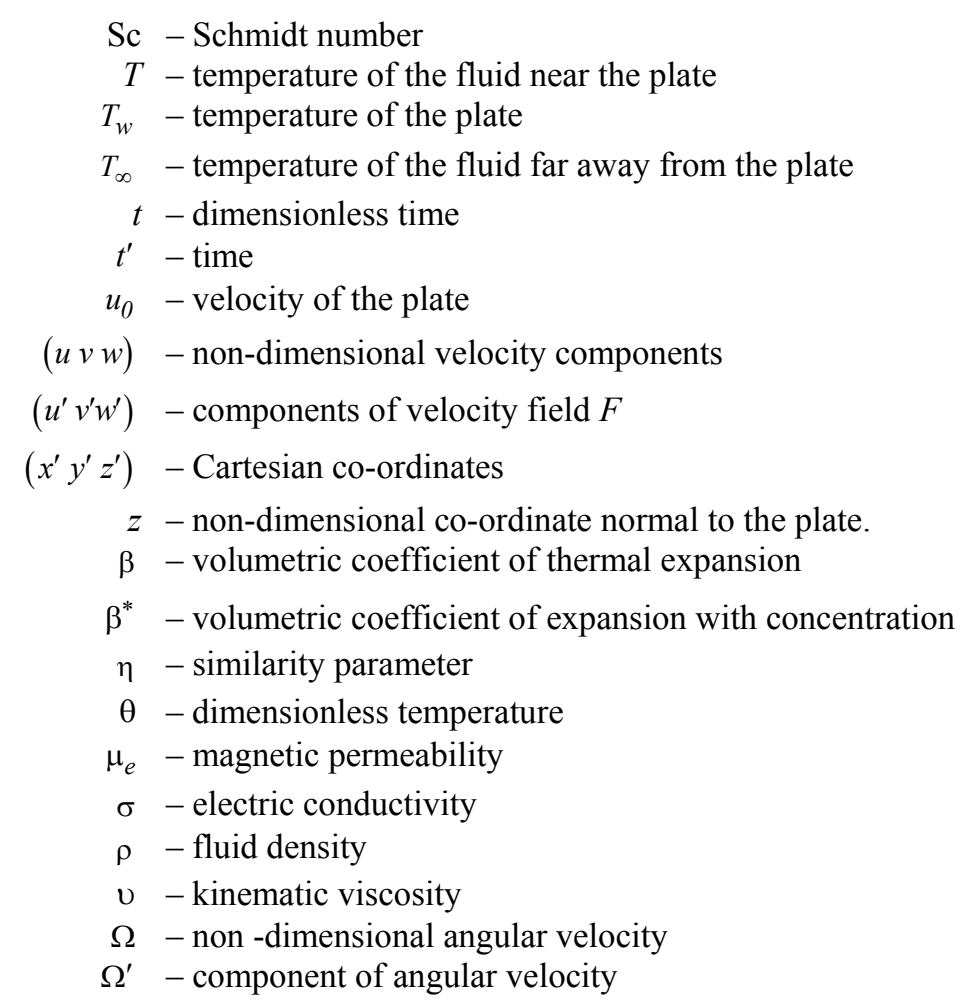

\section{References}

Hayat T. and Abbas Z. (2007): Effects of Hall current and heat transfer on the flow in a porous medium with slip conditions. - Journal of Porous Media, vol.10, No.1, pp.35-50.

Maguna A.N. and Mutua N.M. (2013): Hall current effects on free convection flow and mass transfer past semi-infinite vertical flat plate. - Int. Jnl. of Mathematics and Statistics Studies, vol.1, No.4. pp.1-22.

Muthucumaraswamy R., Sathappan K.E. and Natarajan R. (2008): Heat transfer effects on flow past an exponentially accelerated vertical plate with variable temperature. - Theoretical Applied Mechanics, vol.35, pp.323-331.

Muthucumaraswamy R., Lal T. and Ranganayakulu D. (2011): Rotation effects on MHD flow past an vertical accelerated plate with variable temperature and uniform mass diffusion. - Annlals of Faculty of Engineering Hunedoara Tome IX, Fascicule 1, pp.228-234.

Pop I. (1971): The effect of Hall currents on hydromagnetic flow near an accelerated plate. - J. Math. Phys. Sci. vol.5, pp.375-379.

Raptis A. and Singh A.K. (1981): MHD free convection flow past an accelerated vertical plate. - Letters in Heat and Mass Transfer, vol.8, pp.137-143.

Takhar H.S., Ram P.C. and Singh S.S. (1992): Hall effects on heat and mass transfer flow with variable suction and heat generation. - Astrophysics and Space Science, vol.191, No.1, pp.101-106.

Takhar H.S., Chamkha A.J. and Nath G. (2002): MHD flow over a moving plate in a rotating fluid with a magnetic field, Hall currents and free stream velocity. - Int. J. Engng. Sci., vol.40, No.13. pp.1511-1527.

Watanabe T. and Pop I. (1995): Hall effects on magnetohydrodynamic boundary layer flow over a continuous moving flat plate. - Acta Mechanica, vol.108, pp.35-47.

Received: January 10, 2014

Revised: May 15, 2015 